\title{
Assessment of a Hydraulic Network Model for Zig-zag Cooled Power Transformer Windings
}

\author{
Joris Coddéa, Wim Van der Veken ${ }^{\mathrm{b}}$, Martine Baelmans ${ }^{\mathrm{a}}$ \\ ${ }^{a}$ University of Leuven, Division of Applied Mechanics and Energy Conversion (TME), \\ Celestijnenlaan 300 - bus 2421, 3001 Leuven, Belgium. \\ ${ }^{b}$ CG Power Systems Mechelen, Antwerpsesteenweg 167, 2800 Mechelen, Belgium.
}

\begin{abstract}
The prediction of mass flow distribution is a first, though crucial, step in the thermal design of zig-zag cooled power transformer windings. Typically this prediction is based on thermo-hydraulic network models, which critically depend on the applied correlations. In this paper new correlations for flow through elbows and for dividing/merging flow in T-junctions are numerically extracted from dedicated CFD studies and applied to a case study. It is shown that the new correlations are superior for the prediction of the mass flow distribution for this test case in comparison to correlations thus far available in literature.
\end{abstract}

Keywords: power transformer, oil cooling, pressure drop correlation, T-junction, CFD, network model.

\section{Highlights}

1. Assessment of a hydraulic network model of zig-zag cooled transformer windings

\footnotetext{
*Corresponding author

Email address: joris.codde@kuleuven.be (Joris Coddé)
} 
2. Method: comparison of results with CFD simulations

3. Current pressure drop correlations prove to be inadequate

4. New correlations set up with CFD and built into network model

5. Mass flow distribution and pressure drop over the pass qualitatively well predicted

\section{Introduction}

Thermo-hydraulic network models (THNM) [1] serve already for decades to design oil-cooled power transformer windings. A thermo-hydraulic network model is essentially a one-dimensional model, that describes the average oil velocity as well as oil and coil temperatures throughout transformer windings. It is based on conservation laws complemented with correlations for pressure drop and heat transfer. The application to transformer windings was first detailed by Oliver [1]. More recently, also Campelo et al. [2] calibrated their THNM with Computational Fluid Dynamics (CFD). A notable contribution in this field is the work by Zhang and $\mathrm{Li}[3,4]$, which describes the set-up of their model and subsequently applies it to parametric studies [5]. Rahimpour et al. [6] elaborate another model and also describe the effect of other parameters, including the cooling system height. The ultimate goal is to describe the whole oil circuit in order to accommodate natural circulation. This was achieved by Zhang and Li [7], extending upon their previous work. This is comparable to the later work of Radakovic and Sorgic [8], which is a self-contained description of a closed-loop THNM, with an alternative algorithm for converging the network model evaluation.

An alternative tool for the evaluation of power transformer winding de- 
signs would be a CFD simulation. In this regard there is the interesting work of Torriano et al. $[9,10]$. The first paper [9] zooms in on the required detail of a CFD simulation and the second [10] provides an approach for mapping the results of a $2 \mathrm{D}$ simulation to a $3 \mathrm{D}$ simulation. CFD simulations also enable the study of hot-streaks in the oil, as done by Skillen et al. [11]. The work of Yatsevsky widens the evaluation by including all windings, the tank and the external cooling circuit [12]. Smolka [13] goes one step further by employing a coupled CFD-electromagnetic module with an optimization model. Op. Cit. concerns a dry-type transformer, but the methodology is also applicable to oil-cooled transformer. Nevertheless, while CFD can be very accurate, its use during the design process is too costly both in manpower as in CPU time. While setting up and running a CFD simulation easily needs several hours, network models take a few minutes to evaluate the whole circuit.

The performance of a network model crucially depends on the accuracy of the applied correlations. A traditional source for these correlations are various textbooks, e.g. [14] for hydraulic data and [15] for heat transfer coefficients, which are in turn based on a myriad of experiments. However, their applicability is always limited to specific ranges in dimensions and flow conditions. Most often these conditions limit their applicability for transformer windings, involving laminar oil flow through millimetre-wide channels. On the one hand one could resort to experiments on a replica of a transformer winding [16]. On the other hand, with the advent of CFD, it has become possible to set up valid correlations based on numerical simulations.

This paper focuses on achieving a correct mass flow distribution through the different channels of the transformer windings. This distribution is crucial 
for determining the most important parameter in thermal design, the hotspot temperature.

In the next section a test case is set up to compare the results of the THNM approach with those of CFD simulations. It is shown that the presently available correlations are not suited for the case under consideration. Therefore, more accurate correlations are numerically deduced in the third section. Finally, these correlations are assessed by applying them to the test case.

\section{Nomenclature}

c Arbitrary constant value [-]

$D^{+} \quad$ Dimensionless hydraulic diameter, $D_{\text {rad }} / D_{a x}[-]$

$D_{a x} \quad$ Hydraulic diameter of the axial channel [m]

$D_{\text {rad }}$ Hydraulic diameter of the radial channel [m]

$K \quad$ Pressure drop coefficient, referred to the common branch [-]

$\dot{m} \quad$ Mass flow rate $[\mathrm{kg} / \mathrm{s}]$

$r \quad$ Rounding radius $[\mathrm{m}]$

$r^{+} \quad$ Dimensionless rounding radius, $r / D_{a x}[-]$

Re Reynolds number [-]

$S \quad$ Cross-section $\left[\mathrm{m}^{2}\right]$

$V \quad$ Velocity $[\mathrm{m} / \mathrm{s}]$ 
$\bar{V} \quad$ Average velocity, $\int V d S / S[\mathrm{~m} / \mathrm{s}]$

\section{Greek Symbols}

$\beta \quad$ Mass flow ratio $[-]$

$\Delta P_{C F D}$ Pressure drop obtained via CFD simulation $[\mathrm{Pa}]$

$\Delta P_{f r}$ Frictional pressure drop, defined in Eq. $5[\mathrm{~Pa}]$

$\Delta P_{l o c}$ Local pressure drop, defined in Eq. $4[\mathrm{~Pa}]$

$\Delta P_{\text {pass }}$ Pressure drop over the whole pass $[\mathrm{Pa}]$

$\kappa \quad$ Measure of flow maldistribution, $\max (\dot{m}) / \min (\dot{m})[-]$

$\mu \quad$ Dynamic viscosity $[\mathrm{Pa} \mathrm{s}]$

$\rho \quad$ Density $\left[\mathrm{kg} / \mathrm{m}^{3}\right]$

$\tau_{a n} \quad$ Shear stress as per Eq. $12[\mathrm{~Pa}]$

$\tau_{C F D}$ Shear stress obtained via $\mathrm{CFD}[\mathrm{Pa}]$

$\tau_{f d} \quad$ Shear stress of fully developed flow $[\mathrm{Pa}]$

\section{Subscripts}

- in $\quad$ Property $\bullet$ of the inlet region

- rad Property $\bullet$ of the radial region

- run Property $\bullet$ of the run region 


\section{Case study}

\subsection{Description}

The geometry for this case study (see Figure 1) represents one 'pass' of a transformer winding as an excerpt from a larger zig-zag cooled winding. Hydrodynamically, it is a manifold in a Z-type arrangement [14] with nine parallel channels. Each pass has two axial channels which act as distributor and collector. Radial channels connect distributor and collector. In the context of a network model, each pass can be decomposed into a collection of straight channels, elbows and T-junctions.

Although a winding is rotationally periodic in reality, the geometry is considered two-dimensional. The axial channels (see top view in Figure 2) are characterized by the angle $\Phi$ and

$$
r^{*}=\frac{r_{i}}{r_{o}}=\frac{r_{i}}{r_{i}+W_{a x}} .
$$

For power transformer windings, $r_{i} \approx 0.5 \mathrm{~m}, W_{a x} \approx 6 \mathrm{~mm}$ and $\Phi \approx$ $20^{\circ}$. Thus $r^{*} \approx 1$, which means the axial channels closely resemble parallel plates [17, fig. 75]. The parallel plate assumption is less valid for the radially widening channels. However, as we focus on the determination of the minor losses the radial channels can be treated planar as well. Dimensions are given in Figure 1; the edges of the 'packed' conductors have a rounding radius of 0.8 mm. A hydrodynamically fully developed flow with a mean velocity of 0.485 $\mathrm{m} / \mathrm{s}$ is imposed at the inlet. The inlet and outlet sections are extended in order to generate idealized inlet and outlet conditions. The oil has a density of $856.89 \mathrm{~kg} / \mathrm{m}^{3}$ and a dynamic viscosity of $0.00498 \mathrm{~kg} / \mathrm{ms}$. This leads to a laminar flow governed by $R e=1001$ at the inlet. Thus, the cooling of 


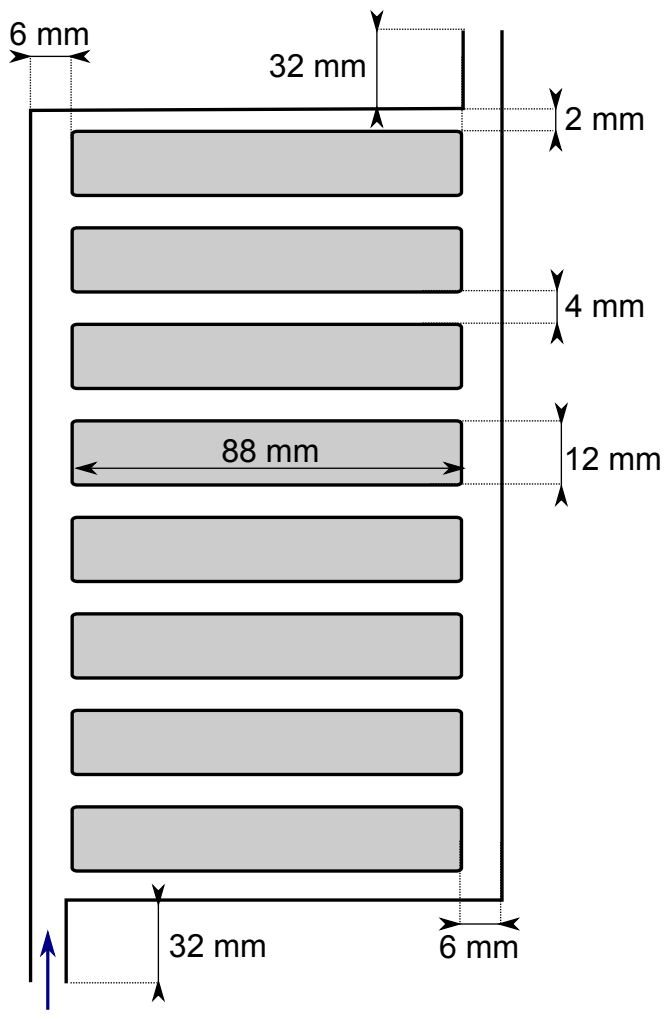

Figure 1: The geometry under study. The oil enters at the bottom left and exits top right. The grey areas are conductors with insulation and impenetrable for the flow.

the winding is in the oil-directed (OD) stage, which is governed by forced convection. Finally, as this study focuses on hydraulic performance, the test case is studied under isothermal conditions.

\section{2. $C F D$ Simulation}

The geometry is meshed with Ansys ICEM. A hybrid mesh with approximately 800,000 elements is created, consisting of 5 layers of rectangles at the boundary and triangles in the core. For the boundary conditions fully developed flow at the inlet and a pressure outlet are assumed. 


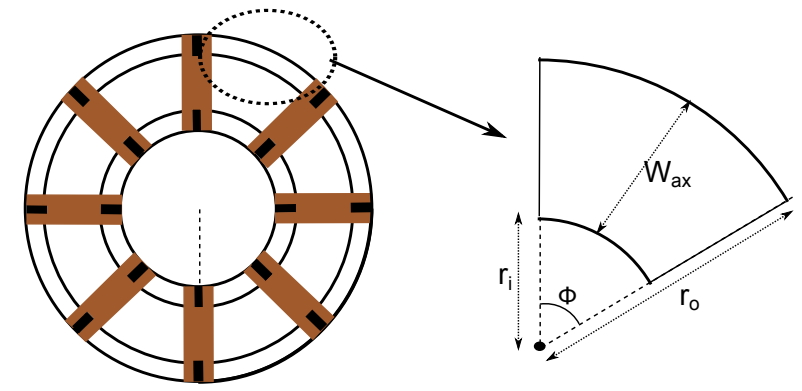

Figure 2: Top view of a winding, of which an axial channel is extracted. The left Figure displays the dimensions of interest of the annular sector duct. This geometry is simplified to parallel plates as laid out in the text.

The problem is solved with Ansys Fluent, using a second order upwind scheme for convective terms of the governing equations, and a second order central scheme for diffusive terms. The convergence criterion is set at $10^{-6}$ for the continuity equation. The global mass imbalance equals $1.1294 \mathrm{e}^{-8}$, relative to the ingoing mass flow. A mesh independence study was executed, with 1.5 times the number of elements of the original mesh. This resulted in variations smaller than 0.5 per mille on the variables of interest.

\subsection{Network model}

The network model of Radakovic and Sorgic [8] is the framework for this approach and guarantees the governing equations for pressure drop and mass conservation:

$$
\begin{gathered}
\sum_{i=1}^{N_{e}} \Delta P_{i}=0 \\
\sum_{j=1}^{N_{f}} \dot{m}_{j}=0
\end{gathered}
$$


are satisfied, where $N_{e}$ is the number of elements composing a closed loop and $N_{f}$ the number of flows entering or leaving a junction. These equations are complemented with pressure drop correlations for each element. For parallel plates, this is fixed at $f=24 / R e$. For splitting and combining flow, the following correlations are selected:

1. The correlations set up by Oliver [1], based on the experimental measurements of Jamison and Villemonte [18]. The experiments were executed on screwed tees with a radius of $1.9 \mathrm{~cm}$ and cover a wide range of conditions. These correlations are applied in a network model for zig-zag cooled windings by Oliver [1], Zhang and Li [3] and Rahimpour et al. [6].

2. The correlations set up by $\mathrm{Wu}$ et al. for circular pipes [19]. Although the flow velocity in the branch region for one of the dividing flow Tjunctions is not covered by the original data, they are selected because $\mathrm{Wu}$ et al. investigated the same cooling configuration.

3. The correlations set up by Winter [20], valid for circular pipes.

For the elbow elements the tabulated data from Idelchik [14] for elbows with sharp corners, a turning angle of $90^{\circ}$ and smooth walls is selected. It is available for various ratios of channel widths, but is set up for turbulent flow conditions $\left(\operatorname{Re}>210^{5}\right)$. This means the Reynolds-number dependency is not incorporated. Nevertheless, Zhang and Li [3] refer to the same correlations.

\subsection{Comparison}

The comparison is performed for mass flow distribution over the parallel channels and for the total pressure drop over the pass. The first measure 


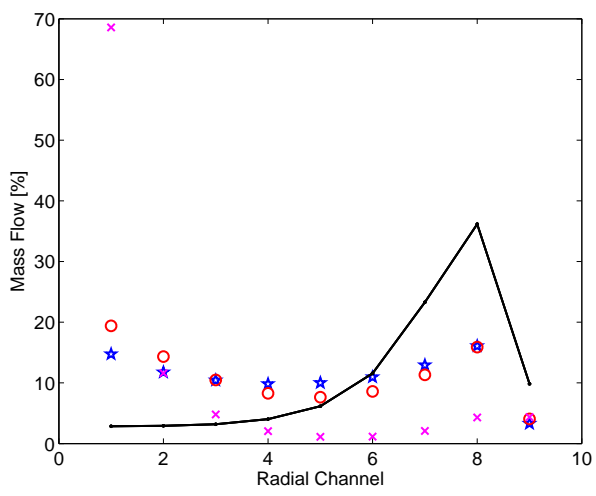

Figure 3: The mass flow distribution for the CFD simulation (-), and the network model equipped with the correlations from Oliver (x), Wu et al. $(\mathcal{\xi})$ and Winter (o).

is important for the thermal transformer design as this is strongly affected by the local flow conditions. It is quantified by the flow maldistribution $\kappa$, which is defined as the ratio of maximum to minimum flow through the parallel channels [21]. The second measure ultimately affects the oil flow through the winding.

The results for the mass flow distribution are depicted in Figure 3. Using the above listed correlations in the THNM gives rise to a too high flow in the first channels. This is explained by the fact that the pressure drop for the straight flow through a dividing T-junction is overestimated. The flow through the first radial channel does not experience this pressure drop. Therefore, the flow in the first channel is too high.

Table 1 contains the data for the flow maldistribution and the pressure drop over the whole pass. It is clear that the correlations of Oliver perform worst. The correlations from $\mathrm{Wu}$ et al. and Winter result in a similar flow distribution with lower flow maldistribution in comparison to the CFD re- 
Table 1: Simulation data.

\begin{tabular}{|l|c|c|}
\hline Correlation & $\kappa$ & $\Delta P_{\text {pass }}[\mathrm{Pa}]$ \\
\hline Oliver & 61.87 & 2143.3 \\
\hline Wu et al. & 4.78 & 230.89 \\
\hline Winter & 4.88 & 163 \\
\hline CFD & 12.64 & 275.35 \\
\hline
\end{tabular}

sults. The overall pressure drop is the closest to the CFD simulation for the correlations of $\mathrm{Wu}$ et al. It is concluded that the existing correlations are inadequate for the thermal design of the transformer winding under considerations. Therefore, new correlations will be extracted in the next section.

\section{Set-up of Pressure Drop Correlations}

To set up new pressure drop correlations, CFD simulations of single Tjunctions are performed. The variable of interest is described in a first subsection. Next, we present a dimensional analysis, which limits the number of simulations needed. The third subsection describes the CFD simulations. Subsequently, the results are presented and correlations are fitted. The procedure to extract pressure drop data from CFD results closely follows the work by El-Shaboury et al. [22], who focused on the heat transfer in 2D dividing T-junctions. The work of Miranda et al. [23] also deals with 2D dividing T-junctions and details the process followed for the simulation. 


\subsection{Extraction of the pressure drop}

The local pressure drop over each element is determined in an artificial geometry. Branches are lengthened - as depicted on Figure 4 - such that the flow can recover to its fully developed regime after the disturbance. The local pressure drop $\Delta P_{l o c}$ is defined by:

$$
\Delta P_{l o c}=\Delta P_{C F D}-\Delta P_{f r}
$$

with $\Delta P_{C F D}$ the measured static pressure drop as assessed by CFD. The expected frictional pressure drop $\Delta P_{f r}$ is equal to:

$$
\Delta P_{f r}=2 \tau_{f d} \frac{L}{D_{h} / 2}
$$

with $\tau_{f d}$ the shear stress of the fully developed flow, and $D_{h}$ the hydraulic diameter $\left(D_{a x}\right.$ or $\left.D_{\text {rad }}\right)$ of the channel. Thus, the local pressure drop accounts for all additional losses caused by the T-element and is artificially localised within the element. This is visualized in Figure 4 by the dotted lines to indicate the different elements.

\subsection{Dimensional Analysis}

We apply a dimensional analysis in order to reduce the number of parameters. The dividing T-element will be used to illustrate the methodology, but the analysis is equally suited to other elements.

In general:

$$
\Delta P_{l o c}=f\left(\bar{V}_{i n}, \bar{V}_{r a d}, \bar{V}_{r u n}, D_{a x}, D_{r a d}, r, \rho, \mu\right)
$$

This functional dependence incorporates already the assumptions made in section 2.1. $\bar{V}_{i n}, D_{a x}$ and $\rho$ are set as the repeating parameters incorporating 


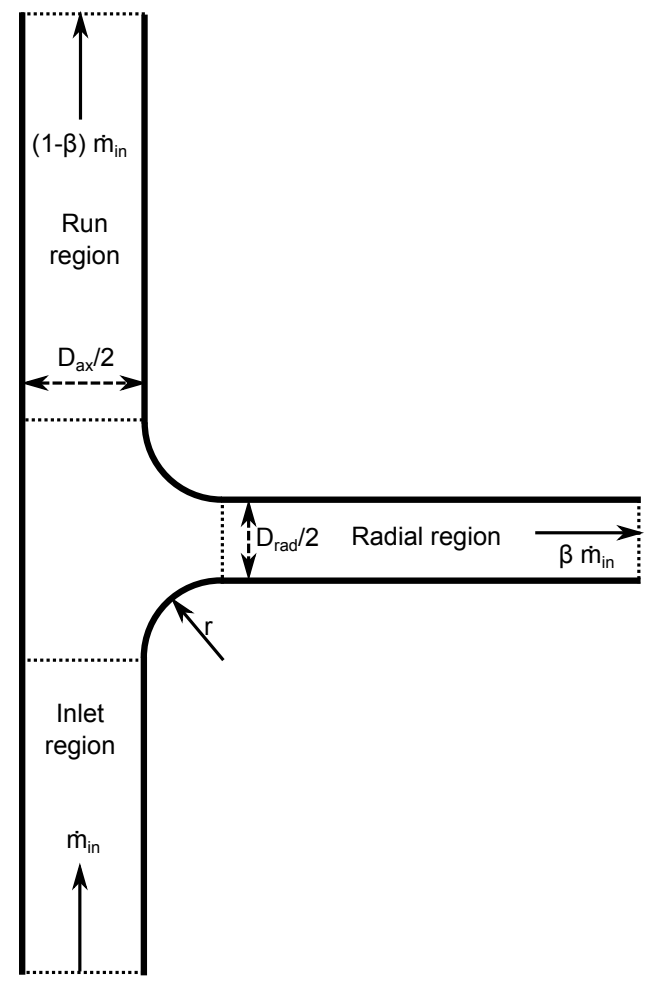

Figure 4: The simulation domain of the T-junction, with terminology and some parameters.

all dimensions present in the full variable set. This results in six dimensionless groups:

$$
K_{\text {in }}=\frac{\Delta P_{\text {loc }}}{\rho \bar{V}_{\text {in }}^{2} / 2}=f\left(\frac{\bar{V}_{\text {rad }}}{\bar{V}_{\text {in }}}, \frac{\bar{V}_{\text {run }}}{\bar{V}_{\text {in }}}, \frac{D_{\text {rad }}}{D_{a x}}, \frac{r}{D_{a x}}, \frac{\rho \bar{V}_{i n} D_{i n}}{\mu}\right)
$$

We now substitute $\frac{\bar{V}_{\text {rad }}}{\bar{V}_{i n}}$ by a product of two dimensionless groups:

$$
\beta_{r a d}=\frac{\bar{V}_{r a d} D_{r a d}}{\bar{V}_{i n} D_{a x}}=\frac{\dot{m}_{r a d}}{\dot{m}_{i n}}
$$

with $\dot{m}=\rho \bar{V} D / 2$ the mass flow per unit length in the third direction. 
Similarly $\beta_{\text {run }}$ is defined. By virtue of the continuity equation:

$$
\dot{m}_{\text {in }}=\dot{m}_{\text {run }}+\dot{m}_{\text {rad }}
$$

we can annihilate the dimensionless group $\beta_{\text {run }}$ :

$$
\beta_{\text {run }}=\frac{\dot{m}_{\text {run }}}{\dot{m}_{\text {in }}}=1-\beta_{\text {rad }}
$$

This results in:

$$
K_{\text {in }}=f\left(\beta_{\text {rad }}, D^{+}, r^{+}, R e_{i n}\right)
$$

with $D^{+} \equiv D_{r a d} / D_{a x}$ the dimensionless ratio of hydraulic diameters, $r^{+} \equiv r / D_{a x}$ the dimensionless rounding radius and $R e \equiv \frac{\rho \bar{V} D_{h}}{\mu}$ the Reynolds number. The range of the parameters can be determined by inserting typical transformer data. The Reynolds number varies from 64 to 1024 with a logarithmic distribution. For $\beta_{\text {rad }}$, the lowest values simulated are 0.02, 0.05 and 0.1 - after which the step size of 0.1 is applied up to $0.9 . D^{+}$and $r^{+}$are set according to the case study, i.e. $1 / 3$ or $2 / 3$ for $D^{+}$and 0.133 for $r^{+}$.

\subsection{CFD Simulations}

The 'measured' pressure drop is determined by CFD simulations. The geometry is meshed with Ansys ICEM. For a rounding radius of 0, a structured mesh is created. For a strictly positive rounding radius, a hybrid mesh is created, consisting of 5 layers of rectangles at the boundary and triangles in the flow core. The height of the first cell is set according to Miranda [23], i.e. $2 \Delta x / D_{h}=0.01$. The length of the cell is always lower than $0.3 \mathrm{~mm}$. The number of cells in the cross-section equals 60 for $D_{h}=6 \mathrm{~mm}$ and 45 for 4 and $2 \mathrm{~mm}$. 
The boundary conditions are fully developed flow at the inlet and pressure outlets at both the outlet and radial branch. One of the branches has a targeted mass flow rate.

Ansys Fluent, involving a second order upwind scheme for convective terms and a second order central scheme for diffusive terms, is used to solve the problem. The convergence criterion is set as low as $10^{-9}$ for the continuity equation in order to guarantee the correct mass flow distribution factor $\beta$.

Additionally, the global mass balance - relative to the total oil flow - is checked to be lower than $10^{-6}$. Furthermore, the frictional pressure drop in the first (for the inlet) or last $10 \mathrm{~mm}$ of the branch (for the outlet channels) is extracted from the wall shear stress as computed in the simulation. It is compared to the analytical shear stress of fully developed flow:

$$
\tau_{a n}=f \frac{\rho \bar{V}^{2}}{2}
$$

with $f=24 / R e$. The absolute percentage error (APE):

$$
A P E_{\tau}=\frac{\left|\tau_{C F D}-\tau_{a n}\right|}{\tau_{a n}}
$$

is aimed at to be smaller than $0.4 \%$. This measure reveals that the mesh is fine enough and that the flow is hydrodynamically fully developed near the inlet and outlet sections. Eventually, $\tau_{C F D}$ is used in Equation 5.

The length of the radial and run regions is an important attribute of the mesh in order to achieve fully developed flow. The length of the regions can be represented nondimensionally:

$$
L^{+}=\frac{L}{D_{h}}
$$


The length is based on the hydraulic entrance length as correlated by Chen [17]:

$$
L_{h y}^{+}=\frac{L_{h y}}{D_{h}}=\frac{0.315}{0.0175 R e+1}+0.011 R e
$$

For $R e=1024, L_{h y}^{+}$is 11.28. Taking $L^{+}$equal to $L_{h y}^{+}$is inadequate, as demonstrated by Tables 2 and 3 - these are valid for $r^{+}=0$ and $R e_{i n}=1024$. As a rule of thumb, $L^{+}$has to be at least two times $L_{h y}^{+}$and larger when $D^{+}$ is either small or large. The length of the inlet region is far less influential and $L_{i n}^{+}$is topped at 5 .

The influence of the number of cells in the cross-section can be appreciated from the third column in Table 3. When the number of elements is multiplied by 1.25 , the APE on $\tau$ lowered by a factor 2 . The table also shows the difference between the pressure drop coefficient based on the shear stress from the CFD simulation $\left(K\left(\tau_{C F D}\right)\right)$ and the one based on the analytical friction correction $\left(K\left(\tau_{a n}\right)\right)$. It is concluded that both are viable options. The same conclusion applies to the dividing T-junctions. In the remainder of the text the results with $\tau_{C F D^{-}}$-correction will be used.

\subsection{Results}

In this section simulation results for $K$ with $D^{+}=2 / 3$ and $r^{+}=0.133$ are summarized.

\subsubsection{Dividing T-junction}

The results for the dividing T-junction are presented in Figures 5a and $5 \mathrm{~b}$.

For the straight flow with a fixed Reynolds number at the inlet, the pressure drop is the largest for the lowest $\beta$ value. With increasing $\beta$, the pressure 
Table 2: Influence of $L^{+}$on dividing T-junctions with $r^{+}=0$ and $R e_{i n}=1024$

\begin{tabular}{|c|c|c|c|c|c|}
\cline { 3 - 6 } \multicolumn{2}{|c|}{} & \multicolumn{2}{c|}{$\beta=0.1$, Straight flow } & \multicolumn{2}{c|}{$\beta=0.9$, Bending flow } \\
\hline$D^{+}$ & $L^{+}$ & $\mathrm{K}$ & $A P E_{\tau, \text { run }}[\%]$ & $\mathrm{K}$ & $\mathrm{APE}_{\tau, \text { rad }}[\%]$ \\
\hline $1 / 3$ & 12 & -0.3696 & 0.53143 & 11.61 & 0.38258 \\
\hline $1 / 3$ & 24 & -0.3646 & 0.0599 & 11.68 & 0.23275 \\
\hline $1 / 3$ & 30 & -0.3635 & 0.14276 & 11.68 & 0.2513 \\
\hline \hline $2 / 3$ & 12 & -0.3559 & 0.64296 & 2.236 & 2.522 \\
\hline $2 / 3$ & 24 & -0.3509 & 0.0305 & 2.289 & 0.417 \\
\hline $2 / 3$ & 30 & -0.3496 & 0.06 & 2.275 & 0.133 \\
\hline
\end{tabular}

drop first decreases and subsequently increases at larger $\beta$ values. The behaviour is very Reynolds-dependent, as the spread on the values increases and the minimum shifts to the right for lower Reynolds numbers. A majority of the pressure drop coefficients is negative, a phenomenon which is explained by Wang [24].

For the flow that bends, the pressure drop coefficient is far less Reynolds dependent. Therefore, only the range 64-256 has been plotted in Figure 5b. The results for higher Reynolds numbers almost coincide. The pressure drop increases for a higher mass flow in the branch region. For a Reynolds number of 256 , the pressure drop coefficient has its minimum at $\beta \approx 0.1$. There's a slight increase towards smaller $\beta$ values, because the recirculation region at the beginning of the branching region is very high, restricting the flow to a very small section. It should be noted that Jamison and Villemonte [18] argued that the pressure drop coefficient for the bending flow is independent 
Table 3: Influence of the mesh for elbows with $r^{+}=0$ and $R e_{i n}=1024$

\begin{tabular}{|c|c|c|c|c|c|}
\hline$D^{+}$ & $L^{+}$ & $\# \mathrm{El}$ & $K\left(\tau_{C F D}\right)$ & $K\left(\tau_{a n}\right)$ & $\mathrm{APE}_{\tau, \text { rad }}[\%]$ \\
\hline $1 / 3$ & 12 & 1 & 1.664 & 1.67 & 0.5 \\
\hline $1 / 3$ & 24 & 1 & 1.675 & 1.6704 & 0.22 \\
\hline $1 / 3$ & 36 & 1 & 1.676 & 1.667 & 0.257 \\
\hline $1 / 3$ & 36 & 1.25 & 1.675 & 1.671 & 0.136 \\
\hline \hline 1 & 12 & 1 & 1.079 & 1.009 & 6.34 \\
\hline 1 & 24 & 1 & 0.9704 & 0.9644 & 0.28 \\
\hline 1 & 36 & 1 & 0.9645 & 0.9622 & 0.07 \\
\hline 1 & 36 & 1.25 & 0.9642 & 0.9628 & 0.047 \\
\hline \hline 3 & 12 & 1 & 0.2541 & -0.4039 & 44.7 \\
\hline 3 & 24 & 1 & -0.6189 & -0.6943 & 2.9 \\
\hline 3 & 36 & 1 & -0.6961 & -0.7152 & 0.25 \\
\hline
\end{tabular}

from the Reynolds number in the branch region. This would be equivalent to a straight line through zero in Figure 5b, as shown in the appendix. However, for our case this is not valid.

\subsubsection{Combining T-junction}

For the T-junction with combining flow, the radial branch acts as an inlet. The run region now carries the total oil flow, which requires a redefinition of $\beta_{\text {rad }}$ :

$$
\beta_{\text {rad }}=\frac{\dot{m}_{\text {rad }}}{\dot{m}_{\text {run }}}
$$




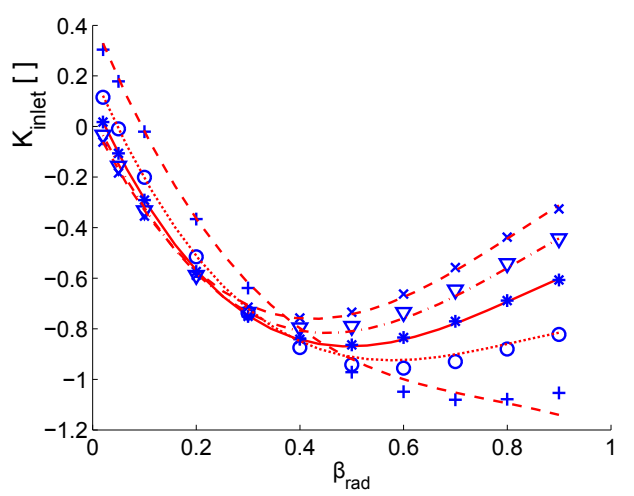

(a) Straight flow

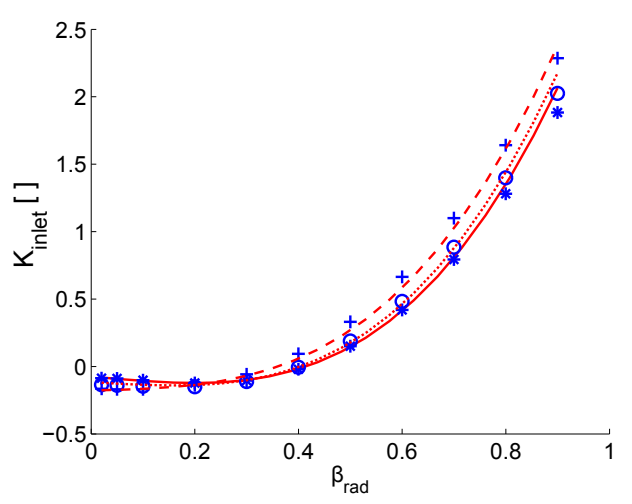

(b) Bending flow

Figure 5: Results for a dividing T-junction with $D^{+}=2 / 3, r^{+}=0.133$ and Reynolds numbers $64(+), 128(\circ), 256(*), 512(\nabla)$ and $1024(x)$. Lines indicate the fit through the data.

The run region also becomes the reference region for $K$ and $R e$ :

$$
K_{\text {run }}=\frac{\Delta P_{\text {loc }}}{\rho \bar{V}_{\text {run }}^{2} / 2}=f\left(\beta_{\text {rad }}, D^{+}, r^{+}, R e_{\text {run }}\right)
$$

The results for $D^{+}=2 / 3$ are presented in Figures $6 \mathrm{a}$ and $6 \mathrm{~b}$. For the straight flow, losses now are the lowest for the smallest $\beta$ values, increase towards a maximum and then decrease for the highest values of $\beta$. The pressure drop coefficient increases for larger Reynolds numbers, and the maximum shifts to higher values of $\beta$. It should be noted that results for $\mathrm{Re}=$ 1024 are not shown as these are very close to the results for $\mathrm{Re}=512$.

Figure $6 \mathrm{~b}$ shows the results for the flow that goes from the branching region to the outlet region. Again, the results for the highest Reynolds number are omitted. The pressure drop coefficient increases from low $\beta$ values towards a maximum at $\beta \approx 0.4$ and decreases towards a minimum at 


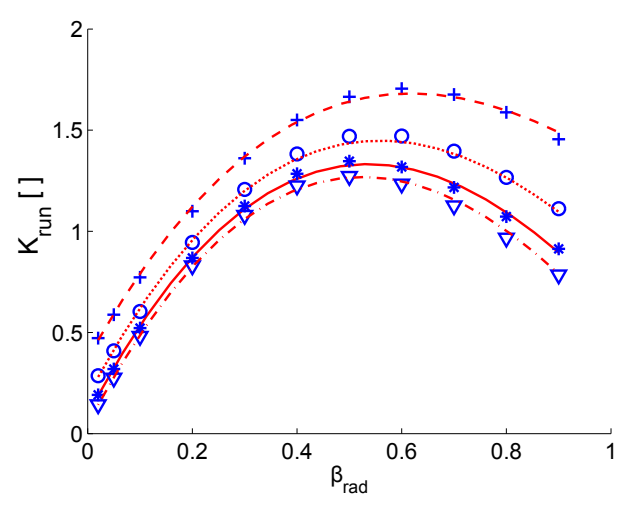

(a) Straight flow

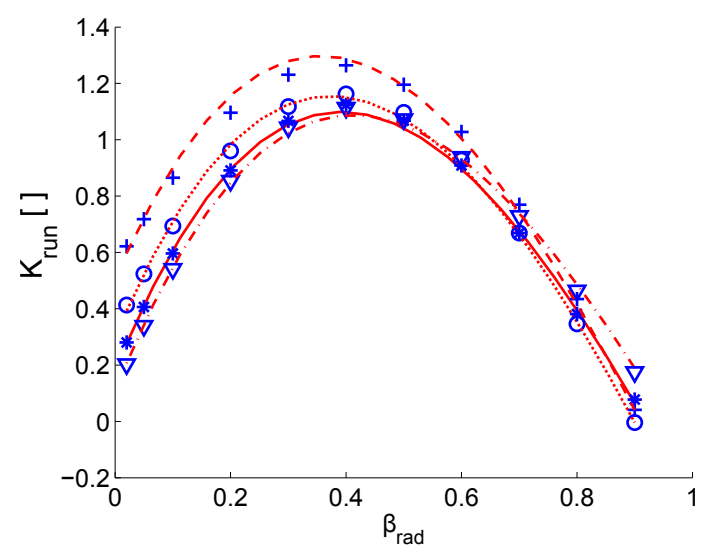

(b) Bending flow

Figure 6: Results for the straight flow in a combining T-junction with $D^{+}=2 / 3, r^{+}=$ 0.133 and Reynolds numbers $64(+), 128(\circ), 256(*)$ and $512(\nabla)$. Lines indicate the fit through the data.

high $\beta$.

The results for the case $D^{+}=1 / 3$, high Re number and low $\beta_{\text {rad }}(i 0.1)$ the last T-junction in the outer channel - are approximately the same as the $D^{+}=2 / 3$ data.

\subsubsection{Elbow}

Applying the terminology of Figure 4 to the elbow element, the junction's outlet region is replaced by a wall. Thus the elbow is independent of $\beta$. For this case, the radial region is chosen as reference region, as the results end up to be scaled better:

$$
K_{\text {rad }}=\frac{\Delta P_{l o c}}{\rho \bar{V}_{\text {rad }}^{2} / 2}=f\left(D^{+}, r^{+}, R e_{r a d}\right)
$$

The range of the dependent parameters was altered to fit the function of 


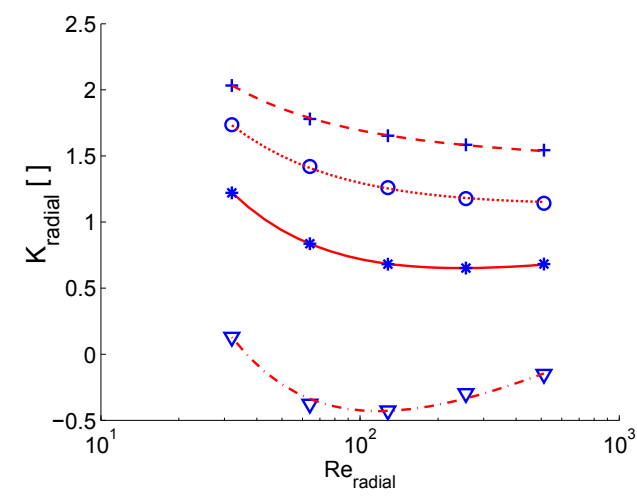

Figure 7: Results for the bending flow in an elbow with $r^{+}=0.133$ and $D^{+} 1 / 3(+), 2 / 3$ $(\circ), 1\left(^{*}\right)$ and $3 / 2(\nabla)$. Lines indicate the fit through the data.

the elbow. Firstly, the elbow element is independent of $\beta$, which allows to select $D^{+}$as the dependent parameter. Secondly, $D^{+}$varies from $1 / 3$ to 3 , as the pressure drop for the inverse ratio also has to be determined. It belongs to the set:

$$
D^{+}=\left\{\frac{1}{3}, \frac{2}{3}, 1, \frac{3}{2}, 2, \frac{5}{2}, 3\right\}
$$

Finally, the elbow never contains the total mass flow: the range of the Reynolds number is lowered to $32-512$.

The results of the simulations are displayed in Figure 7. For clarity, only the results for $D^{+}=[1 / 3,3 / 2]$ are shown. For $D^{+}$smaller than one, the results fit the observations by Edwards et al. [25]: a 1/Re dependence for the lower Reynolds numbers, going towards a constant value for the higher ones. This is not true for $D^{+}>1$, where the results show a minimum. This trend is more pronounced for larger values of $D^{+}$(not shown here). 


\subsection{Fitting}

The results show clear trends. Therefore, it is worthwhile to fit a function through them.

\subsubsection{Functional form}

Badar et al. [26] conducted research on T-junctions of tubes in laminar conditions and opted for

$$
K=a_{1} \ln (R e)+a_{2}
$$

with

$$
a_{i}=b_{2} \beta^{2}+b_{1} \beta+b_{0}
$$

Winter [20], on the other hand, chose for

$$
K=\frac{c_{2} \gamma^{2}+c_{1} \gamma+c_{0}}{R e}
$$

with $\gamma$ the ratio of velocities.

We opt for a combination of both formulae:

$$
K=\frac{a_{0}}{R e}+a_{1} \ln (R e)+a_{2}
$$

Whereby the order of the polynomial for $a_{2}$ has been increased to 3 .

\subsubsection{Fitting procedure}

Because of the large range of $K$ 's, the objective function is chosen to be the relative error:

$$
\min _{a \in \mathbb{R}^{8}}\|F(a)\|_{2}^{2}=\min _{a \in \mathbb{R}^{8}}\left\|\frac{K_{C F D}-K_{\text {corr }}(a)}{K_{C F D}}\right\|_{2}^{2}
$$


In our case, the correlation is linear in the unknown coefficients, such that we can present an analytical solution to this least-squares problem [27]:

$$
\mathbf{a}=\left(\mathbf{X}^{T} \mathbf{W}^{2} \mathbf{X}\right)^{-1} \mathbf{X}^{T} \mathbf{W} \mathbf{y}
$$

with $\mathbf{y}$ the column vector of all loss coefficients $K_{C F D}$, W the diagonal matrix with all weights and $\mathbf{X}$ the matrix of terms which are multiplied by the coefficients in $\mathbf{a}$.

\subsubsection{Evaluation criteria}

The quality of the fittings is evaluated with the following criteria:

$$
\begin{aligned}
S E & =\sum_{i=1}^{s} F(a)_{i}^{2} \\
A R E & =\frac{\sum_{i=1}^{s} F(a)_{i}}{s} \\
M A P E & =\frac{\sum_{i=1}^{s}\left|F(a)_{i}\right|}{s} \\
M A X & =\left\|F(a)_{i}\right\|_{\infty}
\end{aligned}
$$

which are the squared error, the average relative error, the mean absolute percentage error and the maximal error respectively. Additionally, the coefficient of relative determination (CRD) measures the goodness of fit [27]:

$$
C R D=\frac{S S_{\text {reg }}}{S S_{\text {tot }}}
$$

with 


$$
\begin{aligned}
S S_{\text {reg }} & =\frac{\sum_{i=1}^{s}\left(K_{\text {corr }}-\overline{K_{C F D}}\right)^{2}}{\sum_{i=1}^{s} K_{C F D}^{2}} \\
S S_{\text {tot }} & =\frac{\sum_{i=1}^{s}\left(K_{C F D}-\overline{K_{C F D}}\right)^{2}}{\sum_{i=1}^{s} K_{C F D}^{2}}
\end{aligned}
$$

whereby the overbar denotes the mean.

All criteria are reported in Table 4 for the T-junctions and Table 5 for the elbow. Generally, the correlations perform very well, but there are a few outliers. This can be confirmed by visual inspection of Figures 5 to 7 . The reason for the mismatch can be attributed to a combination of the values of the pressure drop coefficients and the use of the relative error: When $\mathrm{K}$ is close to zero, these data points get a very high weight. The maximum deviation between data and correlation will thus concern the lower pressure drop coefficients.

Table 4: Errors for the fittings through the T-junctions: $D^{+}=2 / 3, r^{+}=0.133$ and $R e=[64,1024]$.

\begin{tabular}{|l|c||c|c|c|c|c|}
\hline Element & Flow & CRD & SE & ARE & MAPE [\%] & MAX [\%] \\
\hline \hline \multirow{2}{*}{ Dividing } & Straight & 0.999983 & 0.0542 & 0.001 & 2.42 & 6.06 \\
\cline { 2 - 7 } & Bending & 0.999919 & 0.546 & 0.01 & 7.43 & 37.8 \\
\hline \multirow{2}{*}{ Combining } & Straight & 0.999902 & 0.01 & 0.0002 & 1.3 & 0.025 \\
\cline { 2 - 7 } & Bending & 0.999989 & 0.057 & 0.001 & 2.4 & 14.4 \\
\hline
\end{tabular}


Table 5: Errors for the fitting through the elbow: $D^{+}=[1 / 3,3], r^{+}=0.133$ and $R e=$ $[32,512]$ for the elbow.

\begin{tabular}{|l||c|c|c|c|c|}
\hline Element & CRD & SE & ARE & MAPE [\%] & MAX [\%] \\
\hline \hline Elbow & 0.999840 & 0.045 & 0.0013 & 2.47 & 12.16 \\
\hline
\end{tabular}

\section{Case Study with correlation framework}

The new correlations are now assessed for the case study of section 2 using again the THNM of Radakovic and Sorgic [8] and comparing the THNM results to the CFD results. Correlations in the THNM are calculated onthe-fly based on pre-processed simulation data (in fact the input and output of Equation 11) and fitted for each element. More specifically, for each Tjunction or elbow with Reynolds number $R e$, all cases within $\left[\operatorname{Re} / 4, \operatorname{Re}^{*} 4\right]$ are used for correlation fitting.

The mass flow distribution is presented in Figure 8. The maldistribution factor $\kappa$ is 23.75, almost double of the CFD result, which amounts to 12.64. Contrary to all previous calculations with the network model, the THNM results qualitatively agree well with the CFD simulation. Because the network model underestimates the lowest oil flow and overestimates the highest flow, the maldistribution factor is worse. In the light of hot-spot factor calculation, this situation is still favorable, as the hot-spot will be consistently overpredicted.

The pressure drop through the whole pass is $263.56 \mathrm{~Pa}$, slightly lower than the CFD result, 275.35 Pa. 


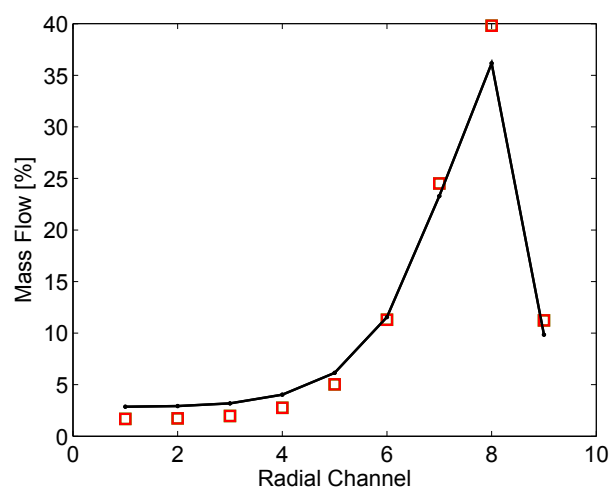

Figure 8: Mass flow distribution over the parallel channels. The black line with dots are for the CFD simulation, the red squares are for the network model.

\section{Conclusion}

In this work considered, THNM is assessed for the hydraulic modelling of zig-zag cooled transformer windings. By comparing THNM with detailed CFD results it is first concluded that THNM with currently available correlations is inadequate. New correlations are therefore numerically derived for all elements in the network model: dividing and combining flow T-junctions and elbows.

The network model of Radakovic and Sorgic [8] equipped with the correlation framework presented here, yielded satisfying results. Both the mass flow distribution over the parallel channels and the pressure drop over the whole pass are qualitatively well predicted and significantly improved in comparison to the formerly used correlations. 


\section{Appendix A. Transformation of pressure drop coefficients}

This section proves the equivalence between pressure drop coefficients with different references. As an example, we concentrate on the pressure drop for the bending flow in a splitting T-junction. Let us suppose that the pressure drop coefficient is referenced to the radial branch and equal to a constant $c$ on the Reynolds number in the branch:

$$
\frac{\Delta P_{\text {loc }}}{\frac{\rho \bar{V}_{\text {rad }}^{2}}{2}}=\frac{c}{R e_{\text {rad }}}
$$

Inserting

$$
\bar{V}_{r a d}=\frac{\beta_{r a d} \bar{V}_{i n} D_{a x}}{D_{\text {rad }}}=\frac{\beta_{r a d} \bar{V}_{i n}}{D^{+}}
$$

and $R e_{\text {rad }}=\beta_{\text {rad }} R e_{i n}$

$$
K=\frac{\beta_{r a d} c}{\left(D^{+}\right)^{2} R e_{i n}}
$$

This is only possible if the dimensions of all three branches are constant.

\section{Acknowledgment}

The authors acknowledge support from the IWT, in the context of a Baekeland Mandate. Simulations were performed on the computing infrastructure of the VSC Flemish Supercomputer Center, funded by the Hercules Foundation and the Flemish Government.

[1] A. Oliver, Estimation of transformer winding temperatures and coolant flows using a general network method, IEE Proceedings C: Generation, Transmission and Distribution 127 (6) (1980) 395-405. doi:10.1049/ipc:19800061. 
[2] H. M. R. Campelo, C. M. Fonte, R. C. Lopes, M. M. Dias, J. C. B. Lopes, Network modelling applied to core power transformers and validation with cfd simulations, in: International Colloquium Transformer Research and Asset Management, 2012.

[3] J. Zhang, X. Li, Coolant flow distribution and pressure loss in ONAN transformer windings-part i: Theory and model development, Power Delivery, IEEE Transactions on 19 (1) (2004) 186-193. doi:10.1109/TPWRD.2003.820225.

[4] J. Zhang, X. Li, Oil cooling for disk-type transformer windings-part I: theory and model development, Power Delivery, IEEE Transactions on 21 (3) (2006) 1318-1325. doi:10.1109/TPWRD.2006.871019.

[5] J. Zhang, X. Li, Oil cooling for disk-type transformer windings-part II: parametric studies of design parameters, Power Delivery, IEEE Transactions on 21 (3) (2006) 1326 -1332. doi:10.1109/TPWRD.2006.871018.

[6] E. Rahimpour, M. Barati, M. Schäfer, An investigation of parameters affecting the temperature rise in windings with zigzag cooling flow path, Applied Thermal Engineering 27 (1112) (2007) 1923 - 1930. doi:http://dx.doi.org/10.1016/j.applthermaleng.2006.12.019. URL http://www.sciencedirect.com/science/article/pii/S1359431107000105

[7] J. Zhang, X. Li, Analysis for oil thermosyphon circulation and winding temperature in ON transformers, in: Power Engineering Society General Meeting, 2007. IEEE, 2007, pp. 1 -8. doi:10.1109/PES.2007.385729. 
[8] Z. Radakovic, M. Sorgic, Basics of detailed thermal-hydraulic model for thermal design of oil power transformers, Power Delivery, IEEE Transactions on 25 (2) (2010) 790 -802. doi:10.1109/TPWRD.2009.2033076.

[9] F. Torriano, M. Chaaban, P. Picher, Numerical study of parameters affecting the temperature distribution in a disc-type transformer winding, Applied Thermal Engineering 30 (14-15) (2010) 2034 - 2044. doi:10.1016/j.applthermaleng.2010.05.004.

URL http://www.sciencedirect.com/science/article/pii/S1359431110002000

[10] F. Torriano, P. Picher, M. Chaaban, Numerical investigation of $3 \mathrm{~d}$ flow and thermal effects in a disc-type transformer winding, Applied Thermal Engineering 40 (0) (2012) 121 - 131. doi:http://dx.doi.org/10.1016/j.applthermaleng.2012.02.011.

URL http://www.sciencedirect.com/science/article/pii/S135943111200107X

[11] A. Skillen, A. Revell, H. Iacovides, W. Wu, Numerical prediction of local hot-spot phenomena in transformer windings, Applied Thermal Engineering 36 (0) (2012) 96 - 105. doi:10.1016/j.applthermaleng.2011.11.054.

URL http://www.sciencedirect.com/science/article/pii/S135943111100682X

[12] V. A. Yatsevsky, Hydrodynamics and heat transfer in cooling channels of oil-filled power transformers with multicoil windings, Applied Thermal Engineering 63 (1) (2014) 347 - 353. doi:http://dx.doi.org/10.1016/j.applthermaleng.2013.10.055.

URL http://www.sciencedirect.com/science/article/pii/S1359431113007746 
[13] J. Smolka, Cfd-based 3-d optimization of the mutual coil configuration for the effective cooling of an electrical transformer, Applied Thermal Engineering 50 (1) (2013) 124 - 133. doi:http://dx.doi.org/10.1016/j.applthermaleng.2012.06.012.

URL http://www.sciencedirect.com/science/article/pii/S1359431112004334

[14] I. E. Idelchik, Handbook of Hydraulic Resistance, 3rd Edition, CRC Press, Boca Raton, Florida, U.S. of America, 1994.

[15] W. M. Rohsenhow, J. P. Hartnett, Y. I. Cho, Handbook of Heat Transfer, 3rd Edition, McGraw-Hill, New York, U. S. of America, 1998.

[16] J. Zhang, X. Li, M. Vance, Experiments and modeling of heat transfer in oil transformer winding with zigzag cooling ducts, Applied Thermal Engineering 28 (1) (2008) 36 - 48. doi:http://dx.doi.org/10.1016/j.applthermaleng.2007.02.012. URL http://www.sciencedirect.com/science/article/pii/S1359431107000865

[17] R. Shah, A. London, Laminar Flow forced Convection in Ducts, Academic Press, New York, U.S. of America, 1978.

[18] D. K. Jamison, J. R. Villemonte, Junction losses in laminar and transitional flows, Journal of the Hydraulics Division 97 (7) (1971) 0045-1063.

[19] W. Wu, Z. Wang, A. Revell, P. Jarman, Computational fluid dynamics calibration for network modelling of transformer cooling flows - part II: pressure loss at junction nodes, Electric Power Applications, IET 6 (1) (2012) 28 -34. doi:10.1049/iet-epa.2011.0005. 
[20] H. Winter, Contribution to the hydraulic branching problem, Ingenieure-Archiv 9 (1955) 239-249, (in German).

[21] G. F. Jones, Heat transfer and flow distribution within radiantconvective finned-tube manifold assemblies, Ph.D. thesis, Pennsylvania Univ., Philadelphia, U.S. of America (1981).

[22] A. M. F. El-Shaboury, H. M. Soliman, S. J. Ormiston, Laminar forced convection in two-dimensional equal-sided and reduced branching ducts, Numerical Heat Transfer, Part A: Applications 42 (5) (2002) 487-512. arXiv:http://www.tandfonline.com/doi/pdf/10.1080/10407780290059666, doi:10.1080/10407780290059666.

URL http: //www . tandf online.com/doi/abs/10 . 1080/10407780290059666

[23] A. I. P. Miranda, P. J. Oliveira, F. T. Pinho, Steady and unsteady laminar flows of Newtonian and generalized Newtonian fluids in a planar T-junction, International Journal for Numerical Methods in Fluids 57 (3) (2008) 295-328. doi:10.1002/fld.1626.

URL http://dx.doi .org/10.1002/fld.1626

[24] J. Wang, Theory of flow distribution in manifolds, Chemical Engineering Journal $168 \quad(3) \quad(2011) \quad 1331 \quad$ - 1345. doi:http://dx.doi.org/10.1016/j.cej.2011.02.050.

URL http://www.sciencedirect.com/science/article/pii/S1385894711002300

[25] M. Edwards, M. Jadallah, R. Smith, Head losses in pipe fittings at low Reynolds numbers, Chemical Engineering Research and Design 63 (1) (1985) 43-50. 
[26] A. Badar, R. Buchholz, Y. Lou, F. Ziegler, CFD based analysis of flow distribution in a coaxial vacuum tube solar collector with laminar flow conditions, International Journal of Energy and Environmental Engineering 3 (1). doi:10.1186/2251-6832-3-24.

URL http://dx.doi.org/10.1186/2251-6832-3-24

[27] C. Tofallis, Least squares percentage regression, Journal of Modern Applied Statistical Methods 7 (2) (2009) 526-534. 